\title{
Bandgap determination of P(VDF-TrFE) copolymer film by electron energy loss spectroscopy
}

\author{
DIPANKAR MANDAL*, K HENKEL ${ }^{\dagger}, K_{\text {MÜLLER }}^{\dagger}$ and D SCHMEIßER ${ }^{\dagger}$ \\ Department of Physics, Jadavpur University, Kolkata 700 032, India \\ ${ }^{\dagger}$ Brandenburgische Technische Universität Cottbus, Angewandte Physik-Sensorik, K.-Wachsmann-Allee 17, 03046 \\ Cottbus, Germany
}

MS received 24 February 2009

\begin{abstract}
The ferroelectric $\beta$ of poly(vinylidene fluoride trifluoroethylene), P(VDF-TrFE) is confirmed for $100 \mathrm{~nm}$ thickness spin coated copolymer film. The homogeneous coverage of the copolymer film is investigated by the help of X-ray photoelectron spectroscopy (XPS). Most importantly, the existing bandgap in the crystalline phase of the copolymer is determined directly from the electron energy loss spectroscopy (EELS).
\end{abstract}

Keywords. Ferroelectric polymer; P(VDF-TrFE) film; $\beta$-phase; bandgap; EELS.

\section{Introduction}

Ferroelectric polymer polyvinylidene fluoride (PVDF) and its copolymer poly(vinylidene fluoride trifluoroethylene) $(\mathrm{P}(\mathrm{VDF}-\mathrm{TrFE}))$ have generated tremendous attention due to their excellent properties for utility value. Over the last thirty years, $\mathrm{P}(\mathrm{VDF}-\mathrm{TrFE})$ is the most studied ferroelectric polymer (Furukawa 1989). It has tremendous applications in electronic industry, such as soft transducers, infrared imaging, and compact capacitors and holds a promising future in the field of non-volatile memory (Auciello et al 1998; Henkel et al 2009). $\mathrm{P}(\mathrm{VDF}-\mathrm{TrFE})$ crystallizes into various crystal forms depending on their molar content ratios of $\operatorname{VDF}(x)$ and TrFE (1-x), and on crystallization conditions (Lando and Doll 1968; Tashiro et al 1984a; Ohigashi 1985). Under ordinary pressure, the $\alpha$ crystal (most common polymorph) form consisting of alternating trans-gauche (TGT $\bar{G})$ molecules packed in antiparallel fashion, is stable for the VDF homopolymer (PVDF), while the $\beta$ phase crystal consisting of all-trans (TTTT) chains becomes more stable on introducing TrFE- $\left(\mathrm{CHF}-\mathrm{CF}_{2}\right)$-sequences into $\mathrm{VDF}-\left(\mathrm{CH}_{2}-\mathrm{CF}_{2}\right)$-chains (Farmer et al 1972). The $\beta$ phase crystal is well known to be of ferroelectric form, revealed by its $D-E$ hysteresis loops (Furukawa et al 1980; Davis et al 1984), polarization switching (Tajitsu et al 1987), temperature behaviour of the dielectric constant (Furukawa et al 1980; Furukawa 1984; Tajitsu et al 1987) and anomalous X-ray dispersion (Takahashi et al 1987). The structural detail of the ferroelectric $\beta$ phase is described by several workers (Lando and Doll 1969; Furukawa 1989;

\footnotetext{
*Author for correspondence (dipankar@phys.jdvu.ac.in)
}

Legrand 1989). Addition of the larger and less molar ratio of TrFE ruins the transition temperature by reducing the average dipole moment of the chains, expanding the lattice, and introducing defects. This ruined ferroelectricity on addition of $\mathrm{TrFE}$ reduces transition temperature (Lovinger et al 1984). In the present investigation, the composition of $70 \% \mathrm{VDF}$ and $30 \% \mathrm{TrFE}$ molar ratio is chosen, because it has the most distinct ferroelectric properties and can be made mostly crystalline without stretching treatments.

There are a few attempts to understand the electronic structure, band dispersions, band symmetries of PVDF and its copolymer (Tashiro et al 1984; Choi et al 1998; Duan et al 2003). However, until now there has been a lack of direct experimental evidence for bandgap estimation of the copolymer. We have employed an experimental technique, electron energy loss spectroscopy (EELS), to estimate the existing bandgap of the P(VDF-TrFE) copolymer, which is expected to have potential influence on material properties.

\section{Experimental}

The $p$ type $\mathrm{Si}(100)$-wafers (Wacker-Chemitronic $\mathrm{GmbH}$ ) were used as main substrate for present investigations. Prior to preparation of polymer spin coated films, pieces of Si-wafers were cleaned through a standard RCA-1 cleaning procedure (Kern 1993). P(VDF-TrFE) $(70: 30)$ films with $9 \mu \mathrm{m}$ thickness were supplied by Piezotech $\mathrm{S}$. A., France. $2 \cdot 5 \%(\mathrm{w} / \mathrm{w})$ copolymer solution was prepared with 2-butanone (AZ-EBR, microchemicals $\mathrm{GmbH}$ ). Spin coating of copolymer on Si-wafer was performed inside glove box under Argon atmosphere. $4000 \mathrm{rpm}$ spinning speed leads to $100 \mathrm{~nm}$ thickness of the copolymer film. 
After spin coating, the film was annealed at $135^{\circ} \mathrm{C}$ for $2 \mathrm{~h}$ to improve the crystallinity of the ferroelectric $\beta$-phase (Furukawa 1989).

Thickness of the spin coated film was determined by profilometer measurements (Taylor Hobbson, Tencor instruments). To confirm the $\beta$ phase, X-ray diffraction (XRD) and Fourier transform infrared (FTIR) spectra were taken. XRD was carried out in a 'XRD 3000 TT' device (Seifert-FPM Ahrendsburg, Germany) in Bragg Brentano geometry. The $\mathrm{Co}-\mathrm{K}_{\alpha}$ radiation $(\lambda=1.7902 \AA)$ is the source of the X-ray. FTIR spectra were recorded with Bio-Rad FTS-60A Spectrometer, equipped with deuterated triglycine sulfate (DTGS) detector and Thermo-Nicolet Impact 410 Spectrometers. Spectra were taken at $4 \mathrm{~cm}^{-1}$ resolution and 100 scans were recorded. Spectroscopic characterization by XPS was performed at room temperature in Omicron-UHV System with hemispherical analyzer (Hoffmann et al 2000). XPS survey scan was taken with $\mathrm{Mg} \mathrm{K} \alpha(1253.6 \mathrm{eV})$ excitation with a base pressure of $3 \times 10^{-9}$ mbar. The electron energy loss spectroscopy (EELS) was carried out with Vacuum Workshop (VSW) Monochromator controlled electron range of $10-300 \mathrm{eV}$ installed in Omicron UHV XPS system with a base pressure better than $1 \times 10^{-9}$ mbar. In EELS experiment, two different analyser pass energies ( $2 \mathrm{eV}$ and $5 \mathrm{eV}$ ) were used.

\section{Results and discussion}

\subsection{Conformation of $\beta$-phase by showing results of XRD and FTIR}

The FTIR spectrum is shown in figure 1. The analysis of FTIR spectra has been carried out for poly(tetrafluoroethylene) (Liang and Kirimm 1956; Hannon et al 1969; Masetti et al 1973) and for PVDF (Tashiro and Kobayashi 1989; Day et al 1992). These studies have proven to be enormously useful to select the vibrations in order to interpret the molecular structure of $\mathrm{P}(\mathrm{VDF}-\mathrm{TrFE})$ copolymer. Due to the large mass of the fluorine atom, most infrared-active vibrations for the copolymer are concentrated in a rather narrow region, $1500-400 \mathrm{~cm}^{-1}$ (figure 1). Several vibrational bands for the copolymer have been assigned to specific conformations by Tashiro et al (1981, 1984b) and Tashiro and Kobayashi (1988, 1989) and these assignments are useful in the present analysis.

In figure 1 , the bands marked with arrows, at $505 \mathrm{~cm}^{-1}$, $845 \mathrm{~cm}^{-1}, \quad 1184 \mathrm{~cm}^{-1}, \quad 1290 \mathrm{~cm}^{-1}$ give evidence for all-trans ferroelectric phase. No indication for alternating trans-gauche conformation is observed. For this phase, a strong absorption feature at 802, 612 and $1196 \mathrm{~cm}^{-1}$ occurs, for example.

From the XRD measurement, it is clear that the $\mathrm{P}(\mathrm{VDF}-\mathrm{TrFE})$ films show the crystalline region with co-existence phase of amorphous region (see figure 2). It is well known that $\mathrm{P}(\mathrm{VDF}-\mathrm{TrFE})$ has ferroelectric crystalline $\beta$-phase embedded in an amorphous matrix, and the $\beta$-phase has quasi-hexagonal close packing with orthorhombic $m m 2$ structure (Bune et al 1998). As shown in figure 2 , the copolymer sample exhibits an intense diffraction peak at $2 \theta=23 \cdot 4^{\circ}$, which is a characteristic diffraction peak of the ferroelectric polar $\beta$-phase of the overlapping (110) and (200) plane reflections (Davis et al 1978; Fernandez et al 1987; Bune et al 1998; Fang et al 2005).

\subsection{XPS survey scan}

The XPS survey scan (figure 3) clearly reveals that the absence of $\mathrm{Si}_{2} p$ core level around $99.3 \mathrm{eV}$ binding energy, confirms the complete coverage of copolymer over the Si-wafer.

Furthermore, the spectrum consists of relatively narrow core-level ( $\mathrm{F} 1 s$ and $\mathrm{C} 1 s$ ) photoelectron peaks, a broad Auger transition peak and a valence band spectrum.

From the XPS spectra, the stoichiometric ratio (in atomic percent) of two elements, $\mathrm{C}$ and $\mathrm{F}$, in the $\mathrm{P}(\mathrm{VDF}-$ TrFE) film is determined. The relevant formula is as follows (Innes 1907):

$$
\frac{n_{\mathrm{C}}}{n_{\mathrm{F}}}=\frac{I_{\mathrm{C}} / S_{\mathrm{C}}}{I_{\mathrm{F}} / S_{\mathrm{F}}},
$$

where $I_{\mathrm{C}}$ and $I_{\mathrm{F}}$ are the peak area of $\mathrm{C} 1 s$ and $\mathrm{F} 1 s$ core level photoelectron peaks, respectively. $S_{\mathrm{C}}$ and $S_{\mathrm{F}}$ are the atomic sensitivity factors of carbon and fluorine, respectively. The calculated composition in atomic percent: $\mathrm{C}: \mathrm{F}=47 \cdot 9: 52 \cdot 1$, directly from XPS analysis, whereas

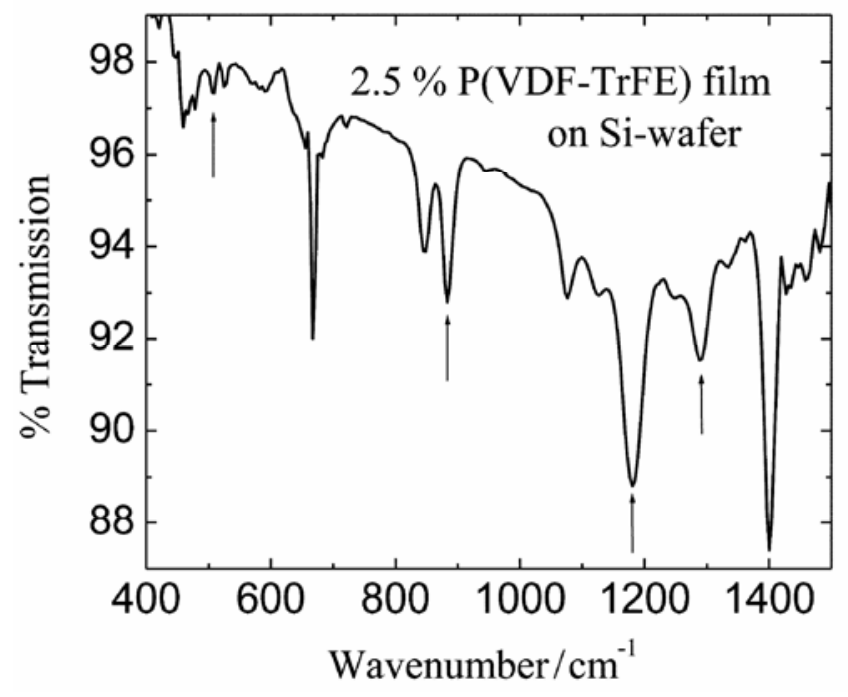

Figure 1. FTIR spectra of a $100 \mathrm{~nm}$ thick spin coated $\mathrm{P}(\mathrm{VDF}-$ TrFE) film on a Si-wafer. The peaks, marked with arrows, correspond to all-trans ferroelectric phase. 
according to a co-polymerization ratio of $\mathrm{VDF}: \mathrm{TrFE}$ $(70: 30)$, the expected theoretical value for the ratio $\mathrm{C}: \mathrm{F}=46 \cdot 5: 53 \cdot 5$. Therefore, an XPS result shows excellent agreement with theoretical calculation.

\subsection{EELS}

By this method, a beam of mono-energetic, low-energy electrons, collimated on a surface, excite the lattice vibrations of the substrate, molecular vibrations of the absorbed species and even electrons. The energy loss of scattered electrons is described by the following equation:

$$
E_{\mathrm{EELS}}=E_{0}-h v \text {, }
$$

where $E_{\mathrm{EELS}}$ is the energy of the scattered electrons, $E_{0}$ the energy of the incident electrons, $h$ the Planck constant and $v$ the frequency of the excited vibration.

One of the most advantageous features of EELS is that it can detect losses in a very broad energy range from infrared to electronic transitions at several electron volts.

A typical energy-loss spectrum shows a zero-loss i.e. elastic peak, constituting electrons that are un-scattered or scattered elastically in the specimen with negligible energy loss. Inelastic scattering is caused by electrostatic interaction between the incident and atomic electrons, and takes a variety of forms. In the case of semiconductor or insulator, excitation of valence electrons to the conduction band gives rise to a spectral component proportional to the joint density of states. The intensity, therefore, remains low up to an energy loss equal to the energy gap, essentially bandgap $\left(E_{\mathrm{g}}\right)$, allowing the latter to be measured. The surface or defect states are visible too.

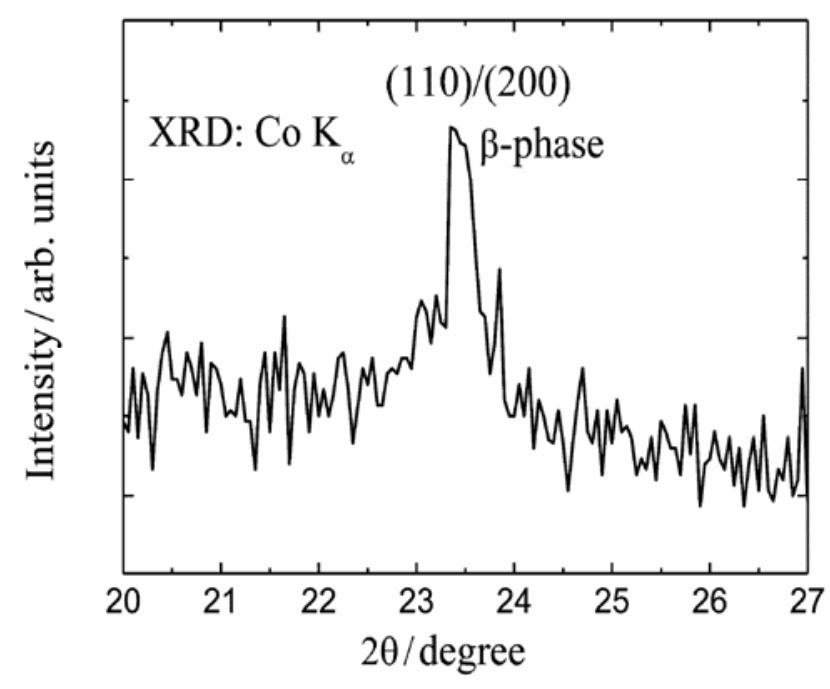

Figure 2. XRD pattern of $100 \mathrm{~nm}$ spin coated film of $\mathrm{P}(\mathrm{VDF}-$ TrFE) on Si-wafer
From FTIR and XRD investigations, it is confirmed that in $100 \mathrm{~nm}$ spin coated copolymer film have all-trans conformation of $\beta$-phase with amorphous surroundings. The theoretical calculations of band structure of PVDF polymer is one of the good points of the present study (Duan et al 2003). The principal of bandgap measurements by the EELS method is described by Kimoto et al (2005).

Figure 4 shows the EELS spectrum of the P(VDF$\mathrm{TrFE}$ ) copolymer film (thickness $\sim 100 \mathrm{~nm}$ ) obtained at an

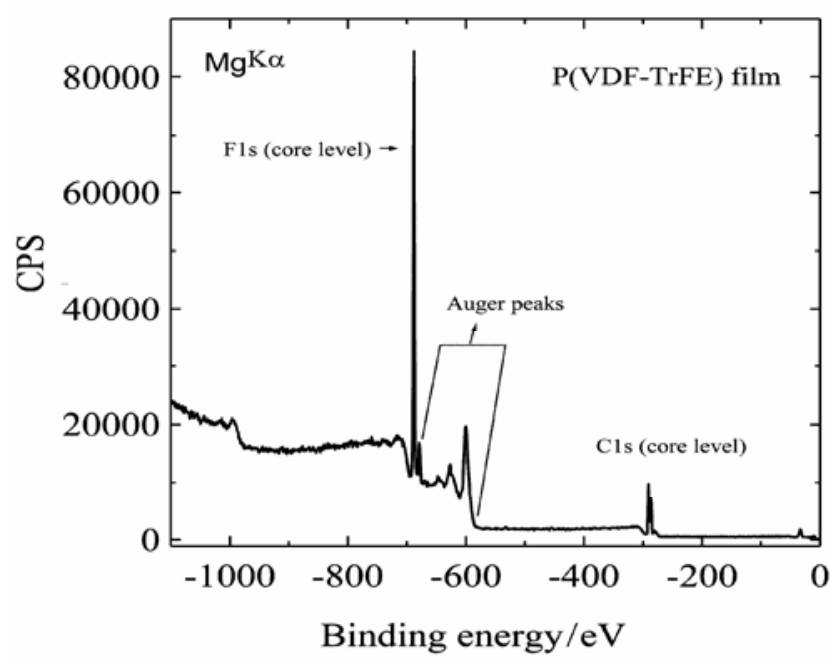

Figure 3. The XPS survey scan of $100 \mathrm{~nm}$ spin coated $\mathrm{P}(\mathrm{VDF}-\mathrm{TrFE})$ copolymer film. The $\mathrm{Mg} \mathrm{K} \alpha(h v=1253.6 \mathrm{eV})$ is used as an X-ray source.

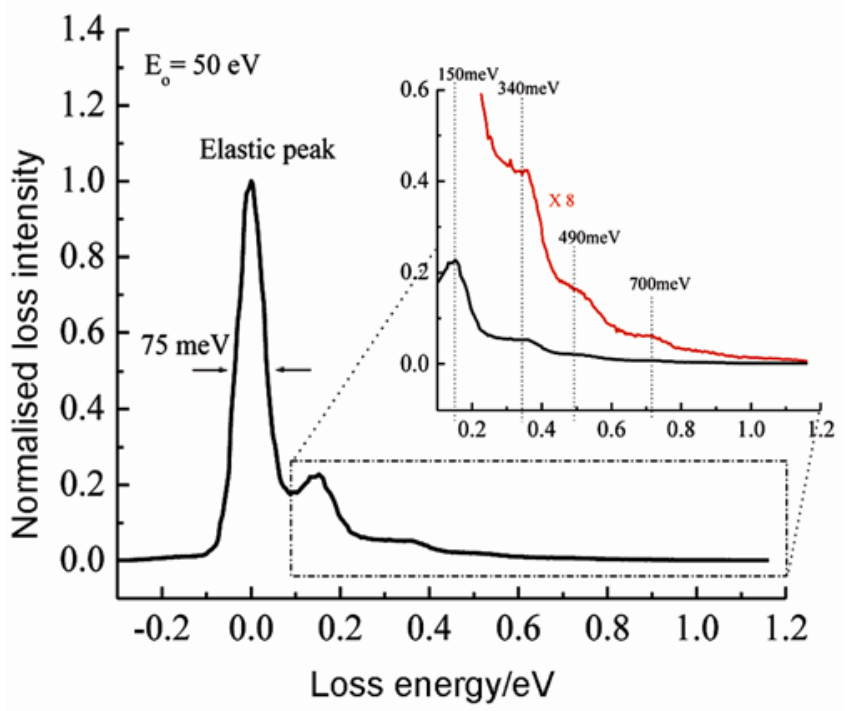

Figure 4. EELS spectrum of a $100 \mathrm{~nm}$ thick P(VDF-TrFE) copolymer film on $\mathrm{Si}(100)$, showing the most intense elastic peak, defect state and vibrational modes. $E_{0}$ denotes the primary electron beam energy and $75 \mathrm{meV}(=\Delta E)$ as instrumental energy resolution. The inset presents the enlarged view of the vibrational modes. 

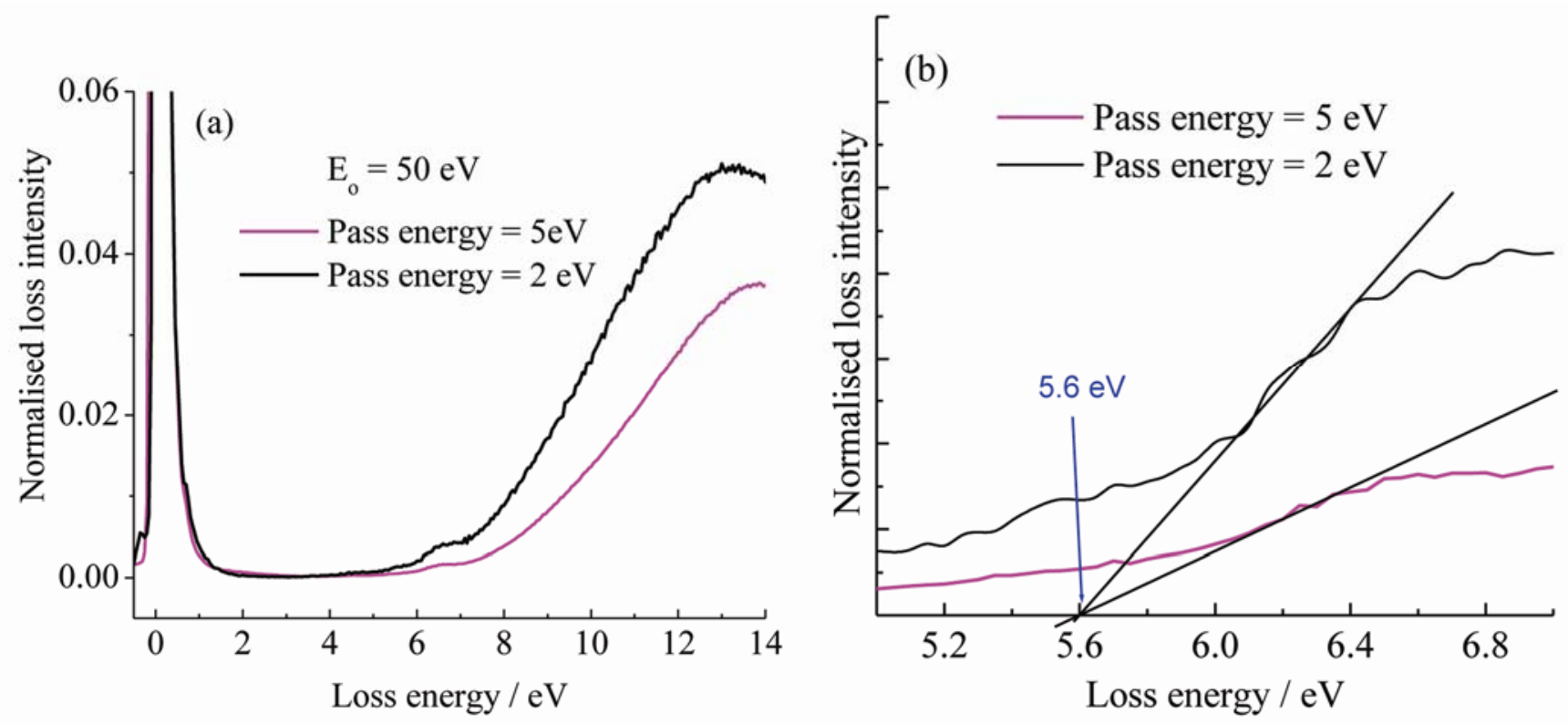

Figure 5. EELS spectrum of a $100 \mathrm{~nm}$ thick film of the $\mathrm{P}(\mathrm{VDF}-\mathrm{TrFE})$ copolymer (a) measured for estimation of the bandgap and (b) enlarged view of the spectrum (a), showing the value of the bandgap $(5 \cdot 6 \mathrm{eV}) . E_{0}$ denotes the primary electron beam energy.

incident primary electron beam energy $\left(E_{0}\right)$ of $50 \mathrm{eV}$ and with a instrumental energy resolution of $75 \mathrm{meV}$. The most intense peak indicates the typical elastic or zero loss peak arising in the EELS spectrum comprising electrons that are un-scattered or scattered elastically in the specimen with negligible energy loss $(E \sim 0)$. The rest of the observable peak appears as shown in the inset of figure 4 , the most intense peak arises due to existence of defects states and the rest of peaks show molecular vibrational modes (Shaw et al 1984).

Figure 5(a) shows the EELS spectrum of extended range of energy loss for bandgap determination for the sample. The bandgap value is estimated by the best linear fit of the loss intensity, extrapolated to loss energy as illustrated by figure $5(\mathrm{~b})$. Therefore, the estimated bandgap $\left(E_{\mathrm{g}}\right)$ value is $5.6 \mathrm{eV}$. The measured $E_{\mathrm{g}}$ value in the present investigation is significant with the band structure model of PVDF crystal (Duan et al 2003).

\section{Conclusions}

The homogeneous $\mathrm{P}(\mathrm{VDF}-\mathrm{TrFE})$ copolymer film with $100 \mathrm{~nm}$ thickness is prepared by spin coated method. The FTIR and XRD results support the semicrystalline behaviour of the film. The existence of the polar ferroelectric $\beta$ phase is confirmed at room temperature for the present film. The evidence of all-trans ferroelectric phase and absence of alternating trans gauche conformation is clearly seen from the FTIR study. Furthermore, XRD results in a distinct diffraction peak which is characteristic of the $\beta$ phase of the overlapping (110) and (200) plane reflections. The calculated amount (in atomic percent) of the $\mathrm{C}$ and $\mathrm{F}$ present in the $\mathrm{P}(\mathrm{VDF}-\mathrm{TrFE})$ film $(\mathrm{C}: \mathrm{F}=47 \cdot 9: 52 \cdot 1)$ is close to the theoretical value $(\mathrm{C}: \mathrm{F}=46 \cdot 5: 53 \cdot 5)$.

Finally, to understand the electronic properties of the copolymer film, EELS results are analysed. The conformation of the defects states and molecular vibrational modes are also conformed by EELS. Furthermore, the direct value of the bandgap $\left(E_{\mathrm{g}}\right)$ in the crystalline phase of the copolymer is determined. The investigated $E_{\mathrm{g}}$ value is $5.6 \mathrm{eV}$. Therefore, the present information in this paper should have realistic impact for the picture of band structure of the copolymer film.

\section{Acknowledgement}

The authors would like to thank Dr Rakesh Sohal (ihp Frankfurt/O, Germany) for his expertise in EELS theory. It is a pleasure to acknowledge the skilful experimental assistance of G Beuckert, P Hoffmann and I Paloumpa.

\section{References}

Auciello Q, Scott J F and Ramesh R 1998 Phys. Today 5122 Bune A V, Fridkin V M, Ducharme S, Blinov L M, Palto S P, Sorokin A V, Yudin S G and Zlatkin A 1998 Nature 391 874

Choi J, Dowben P A, Pebley S, Bune A V, Ducharme S, Fridkin V M, Palto S P and Petukhova N 1998 Phys. Rev. Lett. 80 1328

Davis G T, McKinney J E, Broadhurst M G and Roth S C 1978 J. Appl. Phys. 494998 
Davis G T, Broadhurst M G, Lovinger A J and Furukawa T 1984 Ferroelectrics 5773

Day J A, Lewis E L V and Davis G R 1992 Polymer 331571

Duan C -G, Mei W N, Hardy J R, Ducharme S, Choi J and Dowben P A 2003 Europhys. Lett. 6181

Fang F, Zhang M Z and Huang J F 2005 J. Polym. Sci. Part B: Polym. Phys. 433255

Farmer B L, Hopfinger A J and Lando J B 1972 J. Appl. Phys. 434293

Fernandez M V, Suzuki A and Chiba A 1987 Macromolecules 201806

Furukawa T 1984 Ferroelectrics 5763

Furukawa T 1989 Phase Trans. 18143

Furukawa T, Date M, Fukada E, Tajitsu Y and Chiba A 1980 Jpn J. Appl. Phys. 19 L109

Hannon M J, Boerio F J and Koenig J L 1969 J. Chem. Phys. 50 2829

Henkel K, Lazareva I, Mandal D, Paloumpa I, Müller K, Koval Y, Müller P and Schmeißer D 2009 J. Vac. Sci. Technol. B27 504

Hoffmann P, Mikalo P and Schmeißer D 2000 Solid State Electron. 44837

Innes P D 1907 Proc. R. Soc. A79 442

Kern W (ed.) 1993 Handbook of semiconductor wafer cleaning technology (New Jersey: Noyes) p. 49
Kimoto K, Kothleitner G, Grogger W, Matsui Y and Hofer F 2005 Micron 36185

Lando J B and Doll W W 1968 J. Macromol. Sci. Phys. B 2 205

Legrand J F 1989 Ferroelectrics 91303

Liang C Y and Kirimm S 1956 J. Chem. Phys. 25563

Lovinger J, Johnson G E, Bair H E and Anderson E W $1984 J$. Appl. Phys. 562412

Masetti G, Cabassi F, Morelli G and Zerbi G 1973 Macromolecules 6700

Ohigashi H 1985 Jpn J. Appl. Phys. 2423

Shaw D A, King G C, Cvejanović D and Read F H $1984 J$. Phys. B: Atom. Mol. Phys. 172091

Tajitsu Y, Masuda T and Furukawa T 1987 Jpn J. Appl. Phys. 261749

Takahashi Y, Nakagawa Y, Miyaji H and Asai K 1987 J. Polym. Sci. Part C 25153

Tashiro K and Kobayashi M 1988 Polymer 29426

Tashiro K and Kobayashi M 1989 Phase Trans. 18213

Tashiro K, Takano K, Kobayashi M, Chatani Y and Tadokoro H 1981 Polymer 221312

Tashiro K, Takano K, Kobayashi M, Chatani Y and Tadokoro H 1984a Ferroelectrics 57297

Tashiro K, Takano K, Kobayashi M, Chatani Y and Tadokoro H 1984b Polymer 25195 